\title{
Stage II follow-up on a linkage scan for bipolar disorder in the Ashkenazim provides suggestive evidence for chromosome $12 p$ and the GRIN2B gene
}

Dimitrios Avramopoulos, $M D, P h D^{1,2}$, Virginia K. Lasseter, $B A^{1}$, M. Daniele Fallin, $P h D^{3,4}$, Paula S. Wolyniec, $M A^{1}$, John A. McGrath, MA ${ }^{1}$, Gerald Nestadt, $M D^{1}$, David Valle, $M D^{2}$, and Ann E. Pulver, PhD ${ }^{1,3}$

\begin{abstract}
Purpose: We had previously performed a genome-wide linkage scan for bipolar affective disorder in an Ashkenazi Jewish sample, a population likely to have reduced genetic heterogeneity. This study is a second stage follow-up focusing on regions that showed positive linkage scores in our previous scan but were not fine-mapped at that time. Methods: We genotyped an additional 145 highly polymorphic microsatellites and conducted linkage analyses using standard laboratory and analytical methods. Results: We saw an improvement of the evidence for linkage in most regions, with the most notable change on chromosome 12p13.1-p12.3, where the evidence of linkage is now suggestive. This region harbors the gene encoding the ionotropic glutamate receptor subunit 2B (GRIN2B), a gene that previously yielded evidence for association in a candidate gene study on 323 Ashkenazi Jewish bipolar case-parent trios. We find that the evidence for linkage is significantly correlated with the presence of the putative high-risk allele identified in our candidate gene study. Conclusions: Following up weaker signals can significantly improve linkage signals even after relatively small increases in information content. Our results on chromosome 12p support GRIN2B as a candidate gene for bipolar disorder that needs further investigation. Genet Med 2007: 9(11):745-751.
\end{abstract}

Key Words: glutamate receptors, linkage disequilibrium, polymorphism (genetics), chromosomes, human, pair 12, chromosome mapping, genes, bipolar disorder

Bipolar disorder (BP) is a common, disabling psychiatric disease with a lifetime prevalence reaching 2\% (American Psychiatric Association, 1994). Several types of BP are recognized in the Diagnostic and Statistical Manual, fourth edition that vary in the nature and duration of symptoms and the extent of disability. One well-established diagnostic classification focuses on the intensity of manic symptoms recognizing two categories, BPI and BPII, with BPII showing episodes of less severe mania described as hypomania. Evidence from family, twin, and adoption studies strongly supports a genetic component of BP, with heritability estimates of $60-80 \%^{1}$ and risk to first-degree relatives of 5-10\%. ${ }^{2}$ Although segregation analyses have been inconclusive, they suggest a complex and multigenic susceptibility to $\mathrm{BP}$, involving both genes and environment. Many genome-wide linkage scans and three meta-analyses have been reported, ${ }^{3-13}$ identifying multiple candidate genomic re-

From the ${ }^{I}$ Department of Psychiatry and Behavioral Sciences; ${ }^{2}$ McKusick-Nathans Institute of Genetic Medicine, Johns Hopkins School of Medicine; ${ }^{3}$ Department of Epidemiology; ${ }^{4}$ Department of Biostatistics, Johns Hopkins Bloomberg School of Public Health, Baltimore, Maryland.

Ann E. Pulver, PhD, Johns Hopkins School of Medicine, 1820 Lancaster Street, Suite 300, Baltimore,MD 21231.E-mail: aepulver@jhmi.edu.

Disclosure: The authors declare no conflict of interest.

Submitted for publication June 25, 2007.

Accepted for publication August 7, 2007.

DOI: $10.1097 /$ GIM.0b013e318159a37c gions but with inconsistent replication across studies. One potential reason for this lack of consistency in linkage scan results could be the inconsistency in the definition of the affection status across studies, regarding both the diagnosis and the inclusion of BPI, BPII, and schizoaffective disorder, a condition in which patients present both psychotic and affective symptoms, and which some investigators consider a variant of schizophrenia and others a variant of BP. Another likely reason for the inconsistency in linkage results is the high likelihood of locus heterogeneity, especially if individual genes with small or moderate effects on risk are present or possibly genes that interact are involved. One strategy to reduce locus heterogeneity is to study families from a relatively genetically isolated population that originated from a small number of founders. ${ }^{14,15}$ We recently employed this approach in a genome-wide linkage scan of 41 Ashkenazi Jewish (AJ) families with BP. ${ }^{16}$ More recently we followed the same strategy in an extended sample of $323 \mathrm{AJ}$ case-parent trios, performing a candidate gene association study. ${ }^{17}$

Typically initial genome scans for linkage utilize a marker density of about one marker for every $10 \mathrm{cM}$. Guo and Elston ${ }^{18}$ proposed this approach as the first part of a two-stage design, where the initial low marker density is followed by more dense marker genotyping in genomic regions that show positive results in the first phase. In our original publication we performed a genome scan using one marker every $8.85 \mathrm{cM}$ with 
follow-up of four regions that showed nonparametric linkage (NPL) scores $\geq 2.2$. Here we report a second phase that involves genotyping an additional 145 polymorphic short tandem repeat markers in nine genomic regions (total length 456 $\mathrm{cM}$ on the decode linkage map ${ }^{19}$ ) that originally showed peak NPL scores between 1 and 2.2.

\section{MATERIALS AND METHODS}

The collection of the sample used in this study is described elsewhere. ${ }^{16}$ It comprised 41 families (182 subjects with DNA available) ascertained through a proband with BPI disorder, with at least one additional participating relative with BPI or BPII. All recruitment methods and protocols for the collection of blood samples and clinical data were approved by the Johns Hopkins institutional review board, and appropriate informed consent was obtained from all human subjects. Information about each subject was ascertained from multiple sources including a direct evaluation. Two members of our consensus diagnostic committee (psychiatrists or doctoral-level clinical psychologists) independently reviewed all clinical information ascertained and made judgements about diagnoses and clinical dimensions (see Fallin et al. 2004 ${ }^{16}$ ). We excluded families from these analyses if any grandparent of an affected subject was known to be of non-Ashkenazi descent. Among the total set of 41 families, affected individuals included those with a diagnosis of BPI (76.3\%) or BPII (23.7\%), and this is termed our broad diagnostic model. Twenty-six families had at least two members with BPI. Our narrow diagnostic model included 22 of these families that were informative for linkage when using BPI only as the affected phenotype.

We elected to fine-map all regions that showed a NPL score $\geq 1.0$ in the original $\sim 9 \mathrm{cM}$ scan and that had not been finemapped in our original publication. ${ }^{16}$ These were nine regions on chromosomes 2, 4, 6, 7, 9, 12, 14, 16, and 19. We obtained genotyping through deCODE Genetics (Reykjavik, Iceland, www.decode.com) with markers selected from a list presented by deCODE that included information on marker expected heterozygosities, call rates, and genetic locations under their recent high resolution linkage map. ${ }^{19}$ We selected the most informative set of markers that could be inserted between previously typed markers at even spacing. We genotyped a total of 145 new markers with an average heterozygosity of 0.73 . Every interval was evenly populated with about one marker every 2.4 $\mathrm{cM}$ increasing the density of the initial phase by 4 -fold.

We prepared two 96-well DNA plates (186 DNAs) in our laboratory and sent them to deCODE for genotyping. There, three control individuals from the Centre d'études du Polymorphisme Humain pedigrees were added and the samples were diluted to 15 $\mathrm{ng} / \mu \mathrm{L}$. For each marker the forward primer was fluorescently labeled. Polymerase chain reaction (PCR) amplifications were set up on Zymark ALH 400 (Zymark Corp., Hopkinton, MA), run on MJR Tetrad thermal cycler (Bio-Rad Laboratories, Inc., Waltham, MA) and pooled on Gilson Cyberlab C200 robots (Gilson, Inc., Middleton, WI). Twenty nanograms of genomic DNA were amplified in the presence of 2 pmol of each primer, $0.25 \mathrm{U}$
AmpliTaq Gold, $0.2 \mathrm{mM}$ dinucleotide triphosphate, and $2.5 \mathrm{mM}$ $\mathrm{MgCl}_{2}$ (buffer was supplied by the manufacturer, Applera Corp., Norwalk, CT) in a $5-\mu \mathrm{L}$ reaction. Thermal cycling conditions were denaturing at $95^{\circ} \mathrm{C}$ for 10 minutes, followed by 37 cycles of $94^{\circ} \mathrm{C}$ for 15 seconds, annealing at $55^{\circ} \mathrm{C}$ for 30 seconds, and extension at $72^{\circ} \mathrm{C}$ for 1 minute. The PCR products were supplemented with the internal size standard GS500-LIZ (Applera Corp.), and the pools were separated and detected on ABI-3730 Sequencers (Applera Corp.). Alleles were called automatically using DAC, an allele-calling program developed at deCODE and the program deCODE GT was used to fractionate called genotypes according to quality, and when necessary to edit. Statisticians from deCODE performed quality checks of the genotyping results by examination of plate-panel yield, marker yield, Hardy-Weinberg equilibrium, mismatch error rate, Mendelian error rate, and allele distribution across plates. Genotyping results were delivered to us in a (ASCII) tab-delimited file format including allele calls as binned, but also corresponding calls normalized to Centre d'études du Polymorphisme Humain individual 1347-02 genotypes, a reference sample present in three copies on all plates. An inheritance error report was also provided. Mendelian errors were identified using the PedCheck ${ }^{20}$ and GENEHUNTER ${ }^{21}$ analysis software, and GENEHUNTER was also used to identify markers showing recombination with both their flanking markers. In the first case, the family data for the marker were deleted, whereas in the latter case, marker data for the corresponding individual (the apparent double recombinant) were deleted. The genetic map used was that published by Kong et al. ${ }^{19}$ Allele frequencies were estimated from the parental genotypes and linkage analyses were performed using GENEHUNTER as described in our original genome-wide scan publication. ${ }^{16}$ Genotypes for single nucleotide polymorphism (SNP) rs 1805539 for members of 30 families had been previously acquired as described, ${ }^{17}$ while 25 affected members of the remaining 11 families were genotyped by PCR amplification of genomic DNA using the primers ATCCAGTGGGGAGAGCTAGG and CTGATTCATCCCAGGGCTTC followed by digestion of the 165-bp product with the restriction enzyme MwoI (recognition site: GCNNNNNNNGC) and electrophoresis on a 3\% Nusieve-1\% agarose gel for detection of the resulting fragments of 82 and $83 \mathrm{bp}$ when the $\mathrm{C}$ allele was present in the context GTTTG[G/C]GAAGGGAGC.

\section{RESULTS}

We received data on all 145 markers and all 186 DNAs (no DNA failures), and after all error corrections our call rate was 98.9\% (26,666 successful genotypes of 26,970 attempted). The new markers showed eight instances of double recombination and one Mendelian inconsistency that were considered likely genotyping errors and treated as described in the methods. The average information content in our nine regions rose from 0.8 to 0.86 . Tables 1 and 2 summarize the parametric and nonparametric analysis results for the two diagnostic models. Under the broad model three regions on chromosomes 2, 6, and 19 showed small reductions in NPL scores ranging from -0.07 to -0.11 (total reduction -0.28 NPL points) while the scores 
Table 1

Fine-mapping results for 41 multiplex BPI-BPII Ashkenazi pedigrees

\begin{tabular}{|c|c|c|c|c|c|c|c|c|c|c|c|}
\hline \multirow[b]{2}{*}{ Chromosome } & \multicolumn{5}{|c|}{ Model-free } & \multicolumn{3}{|c|}{ Dominant $^{a}$} & \multicolumn{3}{|c|}{ Recessive $^{b}$} \\
\hline & Loc. (cM) & Peak marker & Info. cont. ${ }^{c}$ & NPL & NPL $\boldsymbol{\Delta}$ or $\downarrow$ & $\mathrm{cM}$ & Max HLOD & $\alpha^{d}$ & $\mathrm{cM}$ & Max HLOD & $\alpha^{d}$ \\
\hline \multicolumn{12}{|l|}{ Chromosome 2} \\
\hline Before fine-mapping & 151 & D2S151 & 0.72 & $2.16(0.016)$ & $\downarrow$ & 153 & 1.54 & 0.61 & 139 & 0.72 & 0.28 \\
\hline Fine-mapping & 155 & D2S151 & 0.86 & $2.05(0.021)$ & & 145 & 1.43 & 0.54 & 184 & 0.46 & 0.18 \\
\hline \multicolumn{12}{|l|}{ Chromosome 4} \\
\hline Before fine-mapping & 31 & D4S419 & 0.74 & $1.89(0.03)$ & $\boldsymbol{\Delta}$ & 34 & 0.71 & 0.43 & 39 & 1.31 & 0.41 \\
\hline Fine-mapping & 40 & D4S3013 & 0.93 & $2.06(0.02)$ & & 39 & 0.86 & 0.39 & 40 & 1.07 & 0.29 \\
\hline \multicolumn{12}{|l|}{ Chromosome 6} \\
\hline Before fine-mapping & 117 & D6S276 & 0.74 & $2.07(0.020)$ & $\downarrow$ & 113 & 1.48 & 0.55 & 23 & 0.51 & 0.21 \\
\hline Fine-mapping & 123 & D6S262 & 0.89 & $1.97(0.025)$ & & 123 & 1.17 & 0.45 & 23 & 0.51 & 0.21 \\
\hline \multicolumn{12}{|l|}{ Chromosome 7} \\
\hline Before fine-mapping & 4 & D7S531 & 0.85 & $1.91(0.028)$ & & 137 & 0.25 & 0.27 & 139 & 0.37 & 0.24 \\
\hline Fine-mapping & 4 & D7S531 & 0.85 & $1.91(0.028)$ & & 140 & 0.19 & 0.2 & 131 & 0.53 & 0.24 \\
\hline \multicolumn{12}{|l|}{ Chromosome 9} \\
\hline Before fine-mapping & 9 & D9S286 & 0.85 & $1.23(0.11)$ & $\boldsymbol{\Delta}$ & 6 & 0.69 & 0.37 & 157 & 1.23 & 0.39 \\
\hline Fine-mapping & 17 & D9S269 & 0.9 & $1.82(0.035)$ & & 10 & 0.87 & 0.37 & 162 & 1.23 & 0.39 \\
\hline \multicolumn{12}{|l|}{ Chromosome 12} \\
\hline Before fine-mapping & 32 & $\mathrm{D} 12 \mathrm{~S} 364$ & 0.87 & $1.08(0.139)$ & $\boldsymbol{\Delta}$ & 35 & 0.38 & 0.3 & 32 & 0.48 & 0.22 \\
\hline Fine-mapping & 33 & D12S373 & 0.85 & $1.8(0.04)$ & & 33 & 0.80 & 0.39 & 27 & $0.84^{e}$ & 0.29 \\
\hline \multicolumn{12}{|l|}{ Chromosome 14} \\
\hline Before fine-mapping & 40 & $\mathrm{D} 14 \mathrm{~S} 288$ & 0.82 & $1.36(0.088)$ & $\boldsymbol{\Delta}$ & 40 & 0.44 & 0.3 & 40 & 0.63 & 0.23 \\
\hline Fine-mapping & 41 & D14S288 & 0.81 & $1.47(0.07)$ & & 41 & 0.49 & 0.31 & 41 & 0.68 & 0.24 \\
\hline \multicolumn{12}{|l|}{ Chromosome 16} \\
\hline Before fine-mapping & 42 & D16S3068 & 0.79 & $1.2(0.116)$ & $\boldsymbol{\Delta}$ & 42 & 0.16 & 0.19 & 38 & 0.72 & 0.27 \\
\hline Fine-mapping & 39 & D16S3068 & 0.79 & $1.47(0.07)$ & & 39 & 0.23 & 0.21 & 39 & 0.73 & 0.26 \\
\hline \multicolumn{12}{|l|}{ Chromosome 19} \\
\hline Before fine-mapping & 29 & D19S226 & 0.81 & $1.34(0.091)$ & $\downarrow$ & 32 & 0.60 & 0.39 & 22 & 0.10 & 0.09 \\
\hline Fine-mapping & 26 & D19S588 & 0.84 & $1.27(0.1)$ & & 34 & 0.55 & 0.4 & 22 & 0.09 & 0.09 \\
\hline
\end{tabular}

${ }^{a}$ Dominant parametric HLODs assumed disease allele frequency $=0.005$, and penetrances $0.65,0.65,0.0096$ for homozygotes, heterozygotes, and noncarriers.

${ }^{b}$ Recessive parametric HLODs assumed disease allele frequency $=0.11$, and penetrances $0.65,0.0096,0.0096$.

${ }^{c}$ Information content at the peak marker.

${ }^{d} \alpha=$ estimated proportion of linked families at this location.

${ }^{e}$ This score between D12S391 and D12S1581.

NPL, nonparametric linkage; HLOD, heterogeneity LOD score; $\mathbf{\Delta}$, increase in NPL score; $\downarrow$, decrease in NPL score.

for chromosome seven remained unchanged. The remaining five regions showed increases in NPL scores ranging from 0.11 to 0.72 (total increase $1.86 \mathrm{NPL}$ points). Similarly, under the narrow model, chromosomes 6,9 , and 19 showed small reductions in NPL scores ranging from -0.04 to -0.08 points (total reduction 0.17 NPL points), while the remaining six showed stronger evidence with increases ranging from 0.05 to 0.68 (total increase 1.61 NPL units). The overall excess and higher magnitude of score increases is encouraging, but the absolute value of the resulting scores remained moderate with the exception of one region on chromosome $12 p$ that improved sig- nificantly under both phenotypic models ( 0.72 and 0.68 NPL units under the broad and narrow model respectively). Most notably under the narrow model (BPI only), the NPL score reached 2.42 and the recessive multipoint heterogeneity LOD score (HLOD) reached 2.05, elevating this region into a "suggestive" result by standard criteria. ${ }^{22}$ Interest in this region was further increased by the results of our recent association study of 64 candidate genes for $\mathrm{BP}$ and schizophrenia in $\mathrm{AJ}$ triads, that had identified the gene glutamate subunit $2 \mathrm{~B}$ (GRIN2B) as being associated with BPI ${ }^{17}$ a finding further supported by an association study reported by Martucci et al. ${ }^{23}$ for a SNP lo- 
Table 2

Fine-mapping results for 22 multiplex BPI-restricted Ashkenazi pedigrees

\begin{tabular}{|c|c|c|c|c|c|c|c|c|c|c|c|}
\hline \multirow[b]{2}{*}{ Chromosome } & \multicolumn{5}{|c|}{ Model-free } & \multicolumn{3}{|c|}{ Dominant $^{a}$} & \multicolumn{3}{|c|}{ Recessive $^{b}$} \\
\hline & $\mathrm{cM}$ & Peak marker & Info. cont. ${ }^{c}$ & NPL $(p)$ & NPL $\boldsymbol{\Delta}$ or $\downarrow$ & $\mathrm{cM}$ & Max HLOD & $\alpha^{d}$ & $\mathrm{cM}$ & Max HLOD & $\alpha^{d}$ \\
\hline \multicolumn{12}{|l|}{ Chromosome 2} \\
\hline Before fine-mapping & 141 & $\mathrm{D} 2 \mathrm{~S} 112$ & 0.72 & $1.85(0.034)$ & $\Delta$ & 143 & 0.89 & 0.64 & 137 & 1.07 & 0.5 \\
\hline Fine-mapping & 132 & $\mathrm{D} 2 \mathrm{~S} 347$ & 0.87 & $1.9(0.03)$ & & 133 & 0.58 & 0.51 & 133 & 0.96 & 0.46 \\
\hline \multicolumn{12}{|l|}{ Chromosome 4} \\
\hline Before fine-mapping & 43 & D4S391 & 0.81 & $1.42(0.079)$ & $\boldsymbol{\Delta}$ & 57 & 0.23 & 0.29 & 36 & 0.48 & 0.38 \\
\hline Fine-mapping & 37 & D4S2620 & 0.91 & $1.86(0.033)$ & & 49 & 0.341 & 0.32 & 40 & 0.375 & 0.25 \\
\hline \multicolumn{12}{|l|}{ Chromosome 6} \\
\hline Before fine-mapping & 164 & D6S1581 & 0.64 & $1.61(0.055)$ & $\downarrow$ & 162 & 0.84 & 0.53 & 154 & 0.7 & 0.36 \\
\hline Fine-mapping & 104 & $\mathrm{D} 6 \mathrm{~S} 278$ & 0.9 & $1.57(0.06)$ & & 173 & 0.83 & 0.49 & 164 & 0.821 & 0.32 \\
\hline \multicolumn{12}{|l|}{ Chromosome 7} \\
\hline Before fine-mapping & 143 & D7S530 & 0.82 & $1.51(0.067)$ & $\Delta$ & 145 & 0.22 & 0.33 & 128 & 0.82 & 0.51 \\
\hline Fine-mapping & 171 & D7S636 & 0.89 & $1.81(0.037)$ & & 171 & 0.45 & 0.47 & 131 & 0.6 & 0.35 \\
\hline \multicolumn{12}{|l|}{ Chromosome 9} \\
\hline Before fine-mapping & 9 & D9S286 & 0.83 & $1.55(0.063)$ & $\downarrow$ & 9 & 0.71 & 0.48 & 157 & 0.88 & 0.45 \\
\hline Fine-mapping & 0 & D9S288 & 0.86 & $1.5(0.069)$ & & 0 & 0.52 & 0.36 & 162 & 0.88 & 0.45 \\
\hline \multicolumn{12}{|l|}{ Chromosome 12} \\
\hline Before fine-mapping & 32 & D12S364 & 0.85 & $1.74(0.0427)$ & $\Delta$ & 35 & 0.57 & 0.47 & 32 & 1.32 & 0.52 \\
\hline Fine-mapping & 33 & D12S373 & 0.83 & $2.42(0.009)$ & & 33 & 1.14 & 0.59 & 28 & $2.05^{e}$ & 0.64 \\
\hline \multicolumn{12}{|l|}{ Chromosome 14} \\
\hline Before fine-mapping & 40 & D14S288 & 0.82 & $1.54(0.064)$ & $\boldsymbol{\Delta}$ & 97 & 0.5 & 0.51 & 40 & 0.85 & 0.43 \\
\hline Fine-mapping & 40 & D14S288 & 0.81 & $1.57(0.06)$ & & 95 & 0.52 & 0.53 & 41 & 0.89 & 0.44 \\
\hline \multicolumn{12}{|l|}{ Chromosome 16} \\
\hline Before fine-mapping & 61 & D16S415 & 0.84 & $1.85(0.034)$ & $\Delta$ & 61 & 0.39 & 0.43 & 61 & 0.57 & 0.35 \\
\hline Fine-mapping & 57 & D16S415 & 0.83 & $1.96(0.03)$ & & 39 & 0.55 & 0.446 & 57 & 0.67 & 0.37 \\
\hline \multicolumn{12}{|l|}{ Chromosome 19} \\
\hline Before fine-mapping & 29 & D19S226 & 0.83 & $2.06(0.021)$ & $\downarrow$ & 37 & 1.2 & 0.81 & 22 & 0.71 & 0.4 \\
\hline Fine-mapping & 26 & D19S588 & 0.86 & $1.98(0.025)$ & & 37 & 1.25 & 0.87 & 22 & 0.67 & 0.38 \\
\hline
\end{tabular}

${ }^{a}$ Dominant parametric HLODs calculated in Genehunter V2.0, assuming disease allele frequency $=0.005$, and penetrances $0.65,0.65,0.0096$ for homozygotes, heterozygotes, and noncarriers.

${ }^{b}$ Recessive parametric HLODs assumed disease allele frequency $=0.11$, and penetrances $0.65,0.0096,0.0096$.

${ }^{c}$ Information Content at the peak marker.

${ }^{d} \alpha=$ estimated proportion of linked families at this location.

${ }^{e}$ This score between D12S391 and D12S1581.

NPL, nonparametric linkage; $\boldsymbol{\Lambda}$, increase in NPL score; $\downarrow$, decrease in NPL score.

cated $\sim 55 \mathrm{~kb}$ from our most significant SNP. GRIN2B is located at 13,186,503 Mb on chromosome 12p (UCSC March 2006 assembly) under our current linkage peak (Fig. 1). Furthermore, markers in GRIN2B have been associated with schizophrenia by many studies and supported by a recent meta-analysis. ${ }^{24}$

This convergence of evidence led us to examine the hypothesis that GRIN2B might be responsible for the observed linkage signal. We first examined whether the recessive genetic model supported by the linkage analysis also improved evidence of association. We did this by analyzing our existing genotype data for the associated SNP (rs1805539) using the Family-Based Association Test method $^{25}$ (http://biosun1.harvard.edu/ fbat/fbat.htm) that allows joint tests for association in the presence of linkage under different genetic models in a family based analysis. Consistently, the recessive model gave the most significant evidence, with a $P$ value of 0.003 (the high-risk allele for SNP rs 1805539 was allele $\mathrm{C}$ in the context GTTTG[G/C]GAAGG). The additive $P$ value was 0.006 , while the dominant model was not significant $(P=0.13)$. We then tested whether linkage was 


\section{Chromosome 12 fine mapping}

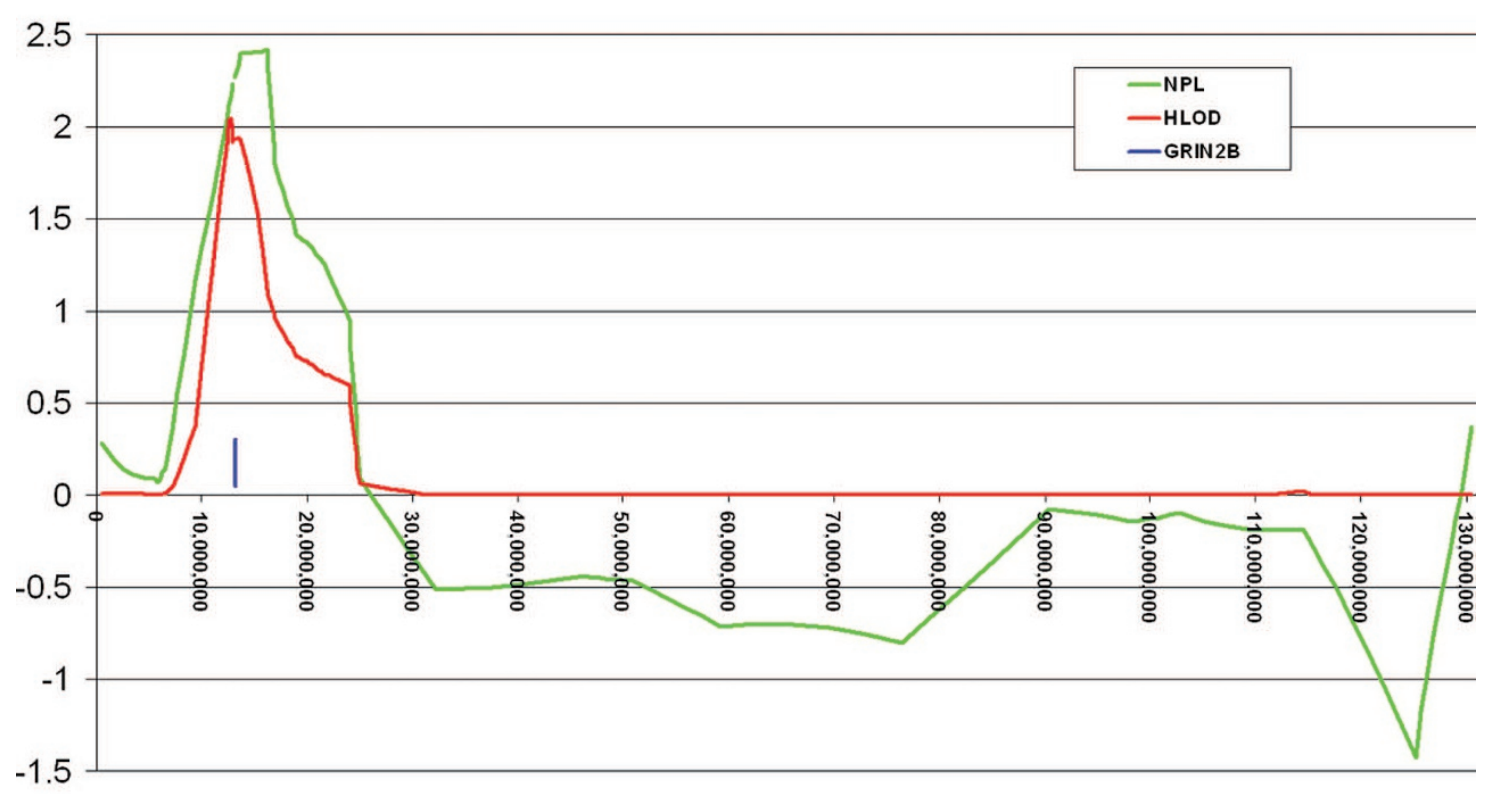

Position (bp) in UCSC March 2006 assembly

Fig. 1. Nonparametric linkage and heterogeneity LOD scores across chromosome 12. The location of GRIN2B is marked by a vertical bar.

Table 3

NPL scores for narrow and broad phenotype model

\begin{tabular}{lccc}
\hline MODEL & Family genotypes & $N$ & NPL \\
\hline Narrow $(N=22)$ & All CC & 8 & 3.15 \\
\multirow{2}{*}{ Broad $(N=41)$} & Not all CC & 14 & 0.62 \\
& All CC & 17 & 2.14 \\
& Not all CC & 24 & 0.24 \\
\hline
\end{tabular}

MODEL, diagnostic model for the definition of affection status; Family genotypes refers to the risk allele "C" of the SNP rs1805539 among affected family members; $N$, number of families; NPL, nonparametric linkage.

stronger in families with homozygotes for this putative highrisk allele by separating the families in two groups; those in which all patients were homozygous for the C allele (group I; 8 and 17 families for the narrow and broad diagnostic models respectively) and the remainder (group II; 14 and 24 families). Under both phenotypic models the group I families showed increased evidence of linkage (Table 3), highest under the narrow phenotypic model consistently with our overall linkage results. This type of stratification of families creates a potential bias as the selection of homozygotes also selects for increased allele sharing. To avoid this bias, we used an analytical method recently described by Li et al. ${ }^{26}$ called Genotype-IBD Sharing Test (GIST). This is a formal method for weighting families according to the members' genotypes and calculating the significance of the correlation between the linkage evidence and a hypothesized high-risk allele. We used the GIST software (http://phg.mc.vanderbilt.edu/content/gist) and a recessive genetic model to examine whether the risk allele correlated with our linkage results, as it would be expected if it accounted for the evidence for linkage according to $\mathrm{Li}$ et al. ${ }^{26}$ The 41 informative pedigrees based on the broad diagnostic definition showed a significant correlation $\left(p_{\text {rec }}=0.02\right)$. The narrow phenotype model identifies only 22 informative families although those carrying the CC genotype showed increased evidence of linkage (Table 3), GIST analysis did not achieve statistical significance. This lack of statistical support despite the observed difference is likely to be due to the very small sample size for this model.

\section{DISCUSSION}

We present the results of a follow-up, fine-mapping study from our genome-wide short tandem repeat scan for genes controlling risk for BP. The original NPL scores of the regions we examined here ranged from 1.08 to 2.16. Although scores of this magnitude are often neglected, we show here that follow-up with a denser set of markers may yield more significant findings. In fact, most regions showed some increase in linkage scores. Moreover, these increases were in aggregate of greater magnitude than the decreases. The results of the fine-mapping performed for our original linkage study further support this observation. We had then fine-mapped four regions and all regions showed improvements in linkage scores (with the exception of chromosome 3 which under the broad phenotype model showed a slight decrease of 0.05 ). Although it is difficult to determine the probability that NPL score would increase by chance alone, it is very encouraging because under the null hypothesis of no true linkage, NPL scores should be equally 
likely to increase or decrease with higher information content. In addition, the fine-mapping of moderate linkage signals allowed us to focus in a new genomic region and a specific gene (GRIN2B) for which we argue that there is now enough converging evidence to warrant further study. In view of these results, this project demonstrates that fine-mapping modest linkage signals might be of value for uncovering remaining linkages from an initial genome-wide screen. The weighing of the cost-benefit of additional fine-mapping, however, is a complicated matter that would need theoretical analyses and require many assumptions.

Our most interesting result was a large increase in NPL and HLOD scores at chromosome 12p12.3, where under the narrow diagnostic model the initial NPL score of 1.7 and the parametric (recessive) HLOD score of 1.32 increased to 2.4 and 2.05 , respectively. The parametric linkage scores now reach the threshold for suggestive linkage. ${ }^{22}$ Although the linkage evidence alone remains below genome-wide significance, it coincides with the location of a gene that provided one of the strongest association signals in our study of candidate genes in a larger AJ sample of BPI patient-parent trios. That sample of 323 trios included cases from 21 of the families used for this linkage analysis, but removing these overlapping families from the association study sample caused no significant change in the result (the previous $P=0.006$ changed to 0.007 ). On the other hand, taking advantage of the partial overlap of these samples and using a method developed for this purpose by Li et al., ${ }^{26}$ we showed the linkage at this locus significantly correlated with the presence of the putative high-risk allele. Li et al. ${ }^{26}$ have shown that a correlation between the presence of a highrisk allele defined from an association study with each family's linkage evidence is not expected under the null hypothesis of no true association, even when the same sample is used for linkage and association. Thus, the GIST result provides additional evidence joining together the linkage and association results supporting the involvement of GRIN2B in BPI. Further independent support is provided by a recent study reporting an association between GRIN2B variants and BP and schizophrenia, ${ }^{23}$ a study by Riley et al. ${ }^{27}$ in southern African Bantuspeaking families that provided weak linkage evidence and a recent linkage study that applied a variance components method on the age at onset of mania ${ }^{28}$ and pointed to marker D12S1292 at 28,992,092 $\mathrm{Mb}$, at the border of our linkage region (Fig. 1).

Despite the converging evidence suggesting that GRIN2B may be associated with high risk for $\mathrm{BP}$, it is important to be cautious in the interpretation of our results. The statistical significance we achieve is at best moderate for both the linkage and its correlation with the putative susceptibility allele. This is especially true if one takes in to account the three genetic and two phenotypic models tested that although highly intercorrelated they still constitute multiple testing; thus, the significance of the reported NPL scores should be appropriately adjusted. The possibility of a false-positive result remains, and this possibility can only be resolved by further replication attempts or by experimentally demonstrating the gene's involvement in
BP. We have mentioned two studies that support this locus for $\mathrm{BP}^{23,27}$; nevertheless, two recently published genome-wide association scans for BP do not show strong significance at this locus. ${ }^{29,30}$ A number of reasons could be responsible for this lack of signal not excluding a false positive result. One obvious alternative explanation is the different ancestry of the studied populations. Our signal comes from an AJ population, while others have reported positive results on a Canadian ${ }^{23}$ and an African ${ }^{27}$ population. The signal from the Canadian sample was relatively weak for BP, and it is hard to assess whether differences of the British and United States samples used in the genome scans might be the reason for not detecting an association above the noise of the many thousands of SNPs examined. When attempting replication for this type of study, it is necessary to minimize differences in study design including the population ancestry. Given the limitations, we mentioned above we believe that attempting such a replication for GRIN2B is very important.

GRIN2B is located in a gene-sparse region with intervals larger than $180 \mathrm{~kb}$ devoid of known genes on either side. Although linkage disequilibrium is slightly more extended in the Ashkenazim, ${ }^{31}$ the nearest gene is beyond the limits of detectable linkage disequilibrium. Thus, although we do not know whether the observed association could be due to variation in GRIN2B, neighboring unknown genes or regulatory elements, the functional relevance of GRIN2B make it the primary candidate and it should be followed up further. GRIN2B encodes a subunit of the NMDA receptor. NMDA receptors are ionotropic glutamate receptors, heteromeric pentameters composed of at least one NR1 subunit and one or more of the four different NR2 subunits: NR2A, NR2B (the product of GRIN2B), NR2C, and NR2D. ${ }^{32}$ The different isoforms of the NR2 subunit give rise to structurally different glutamate receptors in the brain. In addition to glutamate, the NMDA receptor requires allosteric binding of glycine, to a site on the NR1 subunit. The NR2B subunit also has a binding site for polyamines that modulate the functioning of the receptor..$^{33}$ NMDA receptor antagonists, phencyclidine, and ketamine, induce a constellation of behavioral effects in healthy individuals, mimicking the positive, negative, and cognitive symptoms of schizophrenia. ${ }^{34,35}$ Such findings have led to the hypothesis that NMDA receptor-mediated decreases in function may increase the risk for schizophrenia. Support for this hypothesis is provided by studies that have shown differences in NMDA receptor subunits expression patterns in schizophrenic brains $\mathrm{s}^{36,37}$ and by the observation that the GRIN2B subunit increases about 1.2-fold after subchronic administration of the antipsychotic drug clozapine. ${ }^{38}$ DNA variants in and around the GRIN2B gene have been reported to be associated with increased risk of schizophrenia ${ }^{39-41}$ or the effect of clozapine treatment. ${ }^{42,43}$ Moreover, lithium administration reduces the level of NR2B phosphorylation at Tyr1472 and that this is temporally associated with its neuroprotective effect. ${ }^{44}$

An overlap in genetic susceptibility for schizophrenia and BPI has been argued by many investigators and was recently reviewed by Craddock et al. ${ }^{45}$ We agree that there is strong evidence for an overlap of genetic factors contributing to these 
two phenotypes, and we recently published a candidate gene association study ${ }^{17}$ that investigated 440 SNPs in 64 functional and positional candidates for both disorders, genotyped in two sets of triads of AJ descent ascertained for either BPI or schizophrenia. Although at that time studies had suggested GRIN2B only as a candidate for schizophrenia, ${ }^{40,41}$ in our study, it was among the best findings for BPI. More recently, a study by Martucci et al..$^{23}$ also detected an association between GRIN2B DNA variants and both BP and schizophrenia, and a metaanalysis supported the association with schizophrenia. ${ }^{24}$ Our current results provide additional support for the involvement of GRIN2B in BP and indicate that this gene merits further study.

\section{ACKNOWLEDGMENTS}

Supported by National Institutes of Mental Health (NIMH) Grant R01MH58153.

\section{References}

1. Smoller JW, Finn CT. Family, twin, and adoption studies of bipolar disorder. Am J Med Genet C Semin Med Genet 2003;123:48-58.

2. Craddock N, Jones I. Genetics of bipolar disorder. J Med Genet 1999;36:585-594.

3. Blackwood DH, He L, Morris SW, McLean A, et al. A locus for bipolar affective disorder on chromosome 4p. Nat Genet 1996;12:427-430.

4. Detera-Wadleigh SD, Badner JA, Berrettini WH, Yoshikawa T, et al. A high-density genome scan detects evidence for a bipolar-disorder susceptibility locus on 13q32 and other potential loci on 1q32 and 18p11.2. Proc Natl Acad Sci USA 1999;96:56045609 .

5. Morissette J, Villeneuve A, Bordeleau L, Rochette D, et al. Genome-wide search for linkage of bipolar affective disorders in a very large pedigree derived from a homogeneous population in Quebec points to a locus of major effect on chromosome 12q23-q24. Am J Med Genet 1999;88:567-587.

6. Dick DM, Foroud T, Flury L, Bowman ES, et al. Genome-wide linkage analyses of bipolar disorder: a new sample of 250 pedigrees from the National Institute of Mental Health Genetics Initiative. Am J Hum Genet 2003;73:107-114.

7. McInnis MG, Dick DM, Willour VL, Avramopoulos D, et al. Genome-wide scan and conditional analysis in bipolar disorder: evidence for genomic interaction in the National Institute of Mental Health genetics initiative bipolar pedigrees. Biol Psychiatry 2003;54:1265-1273.

8. McInnis MG, Lan TH, Willour VL, McMahon FJ, et al. Genome-wide scan of bipolar disorder in 65 pedigrees: supportive evidence for linkage at $8 \mathrm{q} 24,18 \mathrm{q} 22,4 \mathrm{q} 32$, 2p12, and 13q12. Mol Psychiatry 2003;8:288-298.

9. Middleton FA, Pato MT, Gentile KL, Morley CP, et al. Genomewide linkage analysis of bipolar disorder by use of a high-density single-nucleotide-polymorphism (SNP) genotyping assay: a comparison with microsatellite marker assays and finding of significant linkage to chromosome 6q22. Am J Hum Genet 2004;74:886-897.

10. Sklar P, Pato MT, Kirby A, Petryshen TL, et al. Genome-wide scan in Portuguese Island families identifies $5 \mathrm{q} 31-5 \mathrm{q} 35$ as a susceptibility locus for schizophrenia and psychosis. Mol Psychiatry 2004;9:213-218.

11. Badner JA, Gershon ES. Meta-analysis of whole-genome linkage scans of bipolar disorder and schizophrenia. Mol Psychiatry 2002;7:405-411.

12. McQueen MB, Devlin B, Faraone SV, Nimgaonkar VL, et al. Combined analysis from eleven linkage studies of bipolar disorder provides strong evidence of susceptibility loci on chromosomes 6q and 8q. Am J Hum Genet 2005;77:582-595.

13. Segurado R, Detera-Wadleigh SD, Levinson DF, Lewis CM, et al. Genome scan meta-analysis of schizophrenia and bipolar disorder, Part 3: Bipolar disorder. Am J Hum Genet 2003;73:49-62.

14. Ekelund J, Lichtermann D, Hovatta I, Ellonen P, et al. Genome-wide scan for schizophrenia in the Finnish population: evidence for a locus on chromosome $7 \mathrm{q} 22$. Hum Mol Genet 2000;9:1049-1057.

15. McKusick VA. Genetic studies in American inbred populations with particular reference to the Old Order Amish. Isr J Med Sci 1973;9:1276-1284.

16. Fallin MD, Lasseter VK, Wolyniec PS, McGrath JA, et al. Genomewide linkage scan for bipolar-disorder susceptibility loci among Ashkenazi Jewish families. Am J Hum Genet 2004;75:204-219.

17. Fallin MD, Lasseter VK, Avramopoulos D, Nicodemus KK, et al. Bipolar I disorder and schizophrenia: a 440-single-nucleotide polymorphism screen of 64 candidate genes among Ashkenazi Jewish case-parent trios. Am J Hum Genet 2005;77:918-936.
18. Guo X, Elston RC. One-stage versus two-stage strategies for genome scans. $A d v$ Genet 2001;42:459-471.

19. Kong X, Murphy K, Raj T, He C, et al. A combined linkage-physical map of the human genome. Am J Hum Genet 2004;75:1143-1148.

20. O'Connell JR, Weeks DE. PedCheck: a program for identification of genotype incompatibilities in linkage analysis. Am J Hum Genet 1998;63:259-266.

21. Kruglyak L, Daly MJ, Reeve-Daly MP, Lander ES. Parametric and nonparametric linkage analysis: a unified multipoint approach. Am J Hum Genet 1996;58:13471363.

22. Lander E, Kruglyak L. Genetic dissection of complex traits: guidelines for interpreting and reporting linkage results. Nat Genet 1995;11:241-247.

23. Martucci L, Wong AH, De Luca V, Likhodi O, et al. N-methyl-d-aspartate receptor NR2B subunit gene GRIN2B in schizophrenia and bipolar disorder: polymorphisms and mRNA levels. Schizophr Res 2006;84:214-221.

24. Li D, He L. Association study between the NMDA receptor 2B subunit gene (GRIN2B) and schizophrenia: a HuGE review and meta-analysis. Genet Med 2007; $9: 4-8$.

25. Lange C, Silverman EK, Xu X, Weiss ST, et al. A multivariate family-based association test using generalized estimating equations: FBAT-GEE. Biostatistics 2003;4: 195-206.

26. Li C, Scott LJ, Boehnke M. Assessing whether an allele can account in part for a linkage signal: the Genotype-IBD Sharing Test (GIST). Am J Hum Genet 2004;74: $418-431$.

27. Riley BP, Tahir E, Rajagopalan S, Mogudi-Carter M, et al. A linkage study of the $\mathrm{N}$-methyl-D-aspartate receptor subunit gene loci and schizophrenia in southern African Bantu-speaking families. Psychiatr Genet 1997;7:57-74.

28. Faraone SV, Glatt SJ, Su J, Tsuang MT. Three potential susceptibility loci shown by a genome-wide scan for regions influencing the age at onset of mania. Am J Psychiatry 2004;161:625-630.

29. Genome-wide association study of 14,000 cases of seven common diseases and 3,000 shared controls. Nature 2007;447:661-768.

30. Baum AE, Akula N, Cabanero M, Cardona I, et al. A genome-wide association study implicates diacylglycerol kinase eta (DGKH) and several other genes in the etiology of bipolar disorder. Mol Psychiatry 2007. Epub ahead of print.

31. Shifman S, Kuypers J, Kokoris M, Yakir B, et al. Linkage disequilibrium patterns of the human genome across populations. Hum Mol Genet 2003;12:771-776.

32. Monyer H, Sprengel R, Schoepfer R, Herb A, et al. Heteromeric NMDA receptors: molecular and functional distinction of subtypes. Science 1992;256:1217-1221.

33. Yamakura T, Shimoji K. Subunit- and site-specific pharmacology of the NMDA receptor channel. Prog Neurobiol 1999;59:279-298.

34. Halberstadt AL. The phencyclidine-glutamate model of schizophrenia. Clin Neuropharmacol 1995; 18:237-249.

35. Malhotra AK, Pinals DA, Weingartner $\mathrm{H}$, Sirocco K, et al. NMDA receptor function and human cognition: the effects of ketamine in healthy volunteers. Neuropsychopharmacology 1996;14:301-307.

36. Akbarian S, Sucher NJ, Bradley D, Tafazzoli A, et al. Selective alterations in gene expression for NMDA receptor subunits in prefrontal cortex of schizophrenics. J Neurosci 1996;16:19-30.

37. Grimwood S, Slater P, Deakin JF, Hutson PH. NR2B-containing NMDA receptors are up-regulated in temporal cortex in schizophrenia. Neuroreport 1999;10:461465.

38. Meshul CK, Bunker GL, Mason JN, Allen C, et al. Effects of subchronic clozapine and haloperidol on striatal glutamatergic synapses. J Neurochem 1996;67:1965-1973.

39. Di Maria E, Gulli R, Begni S, De Luca A, et al. Variations in the NMDA receptor subunit 2B gene (GRIN2B) and schizophrenia: a case-control study. Am J Med Genet B Neuropsychiatr Genet 2004;128:27-29.

40. Miyatake R, Furukawa A, Suwaki H. Identification of a novel variant of the human NR2B gene promoter region and its possible association with schizophrenia. Mol Psychiatry 2002;7:1101-1106.

41. Ohtsuki T, Sakurai K, Dou H, Toru M, et al. Mutation analysis of the NMDAR2B (GRIN2B) gene in schizophrenia. Mol Psychiatry 2001;6:211-216.

42. Chiu HJ, Wang YC, Liou YJ, Lai IC, et al. Association analysis of the genetic variants of the N-methyl D-aspartate receptor subunit $2 \mathrm{~b}(\mathrm{NR} 2 \mathrm{~b})$ and treatment-refractory schizophrenia in the Chinese. Neuropsychobiology 2003;47:178-181.

43. Hong CJ, Yu YW, Lin CH, Cheng CY, et al. Association analysis for NMDA receptor subunit $2 \mathrm{~B}$ (GRIN2B) genetic variants and psychopathology and clozapine response in schizophrenia. Psychiatr Genet 2001;11:219-222.

44. Hashimoto R, Hough C, Nakazawa T, Yamamoto T, et al. Lithium protection against glutamate excitotoxicity in rat cerebral cortical neurons: involvement of NMDA receptor inhibition possibly by decreasing NR2B tyrosine phosphorylation. J Neurochem 2002;80:589-597.

45. Craddock N, O’Donovan MC, Owen MJ. Genes for schizophrenia and bipolar disorder? Implications for psychiatric nosology. Schizophr Bull 2006;32:9-16. 